\title{
Analysis of Polarization Mode Dispersion in Fibers and its Mitigation using an Optical Compensation Technique
}

\author{
R. Udayakumar ${ }^{*}$, V. Khanaa ${ }^{2}$ and T. Saravanan ${ }^{3}$ \\ 'Department of Information Technology, Bharath University, Chennai-600073; ruskumar2007@hotmail.com \\ 2Department of Information Technology, Bharath University, Chennai-600073; drvkannan62@yahoo.com \\ ${ }^{3}$ Professor \& Head, Department of ETC, Bharath University, Chennai-600073, India; \\ saravanan.etc@bharathuniv.ac.in
}

\begin{abstract}
The maximum channel density for digital transmission can be achieved by increase the bit rate. When the bit rate cross the $2.5 \mathrm{Gbps}$, the PMD degrade the transmission characteristics. PMD occurs in single mode fiber and because of PMD it impossible to transmit data reliability at high speed. This effects results the widening the pulses and reduce the capacity of transmission of the fiber. This work analyzes the effect of PMD in optical fiber in one hindered km link fiber. The eye diagram, $Q$ value and bit rate for analyzing the PMD. Also an optical compensation technique by which the effect of PMD can be reduced is also analyzed. bit error rate can be achieved by simulating with OptSim 5.3, which includes the algorithms to guarantee the maximum possible accuracy and correct results.
\end{abstract}

Keywords: Local Area Network (LAN), Wavelength Multiplexing (WDM), Principle Rate of Polarization (PSP), Optical Fiber Communication (OFC).

\section{Introduction}

For high speed communication with high quality Optical fiber communication acts as major role. The copper wire between the core replaced by Optical Fiber Communication for telecommunication. Ninety percentage of communication linked by Optical Fiber communication remaining percentage communicated by some other channels. Optical Fiber Communication used not only telecommunication field bit also internet and Local Area network. The advantage of Optical Fiber Communication is low data loss, amplifier kept between long distance, high data carrying capacity. As number of channel increases, dispersion began to show up as obstacles [1]. PMD effects occur only in single mode fiber the resulting the PMD limit the transmission capacity of fiber. The

${ }^{*}$ Corresponding author:

R. Udayakumar (ruskumar2007@hotmail.com)
PMD of installed fiber fluctuates with temperature and stress changes. So, adaptive PMD is needed for Optical Fiber Communication. The resulting the PMD limits the transmission capacity.

PMD which is a time changing quantity is a problem that reduces distances and data rates in a single-mode OFC system.

In general, the effect of PMD on an optical fiber communication link at different bit rates shows that it causes several undesirable effects and it can be compensated through various compensation techniques [2]. The goal of this work is to analyze the effect Polarization Mode Dispersion and its compensation.

The effect of PMD on a fiber link and an optical compensation method is also proposed. By which the 
degradation due to PMD can be mitigated to a certain extent. The concept of optical compensation method is to divide the optical signal that exits from the optical fiber into two and sent to two polarizer [3]. This differential delay cancels the differential group delay caused due to the effect of PMD. And they are complementary to each other to obtain two polarization states. A suitable delay is provided to one of the polarization states and no delay to the other state. Finally the two signals are added and as a result the two components experience a differential delay.

\section{System Modeling}

In high speed transmission PMD will occur and its broadening the pulses and cause to inter symbol interference. Compensation can be achieved by electronic equalization or by using dispersion compwwwwwwensating fibers or by optical equalization. PMD is a physical phenomenon in OFC that causes light pulses to spread. PMD severely reduce the system performance[4]. The PMD is due to geometrical variations in concentricity or ellipticity of fiber core or cladding during manufacture. Therefore in this work an optical compensation method is proposed to compensate for the effect of PMD Thus PMD is varying randomly along the fiber length and therefore the effect of PMD can be reduced by introducing better fiber manufacturing techniques or by compensating for PMD effects [5]. Electronic compensation is not sensitive to the phase and polarization information of the received signal and also it is bit rate dependent so optical compensation is advantageous compared to electronic compensation.

\subsection{Basic Block Diagram}

The block diagram of the work is shown in the Figure 1 and 2. It describes the basic components used for the analysis of the effect of PMD and its compensation [6].

In Figure 1 the effect of PMD in a fiber optic link is analyzed using an emulator and in Figure 2 the optical

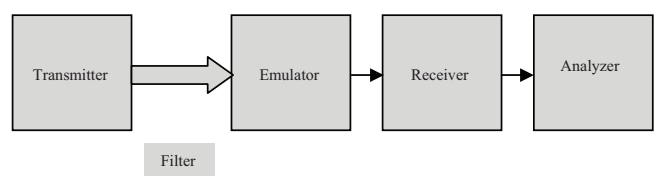

Figure 1. Block diagram for analyzing effect of PMD.

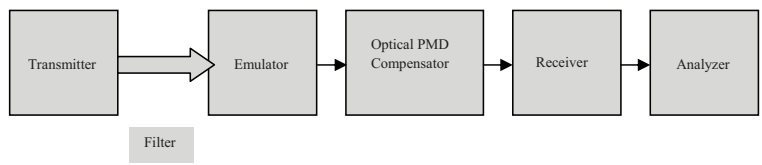

Figure 2. Block diagram for optical PMD compensation.

compensation technique of using an emulator and a deterministic differential group delay is analyzed. An emulator is used after the fiber link to recreate the behavior of an optical field fiber in the laboratory.

\subsection{Simulation Block Diagram}

Amplitude modulator modulates the signal. Then the signal is passed through the fiber channel of $100 \mathrm{~km}$. As the signal travels through the fiber the effect of PMD affects the transmitted.

The simulation is done in OptSim which is one of the costliest simulation software used for optical communication. The below setup shown in Figure 3 simulates PMD effects with and without compensation [7]. The compensation is done by using a Deterministic Differential Group Delay. The effect can be analyzed through eye diagrams and Q estimator.

The light carrier is generated by Lorentzian laser source at the $1550 \mathrm{~nm}$ wavelength. The light can be modulated by the i/p signal by passing through the amplitude modulator The transmitted signal is formed by modulating the light carrier by the NRZ data source.

signal. This delay provided negates the distorted signal through the emulator and thus compensation of PMD is

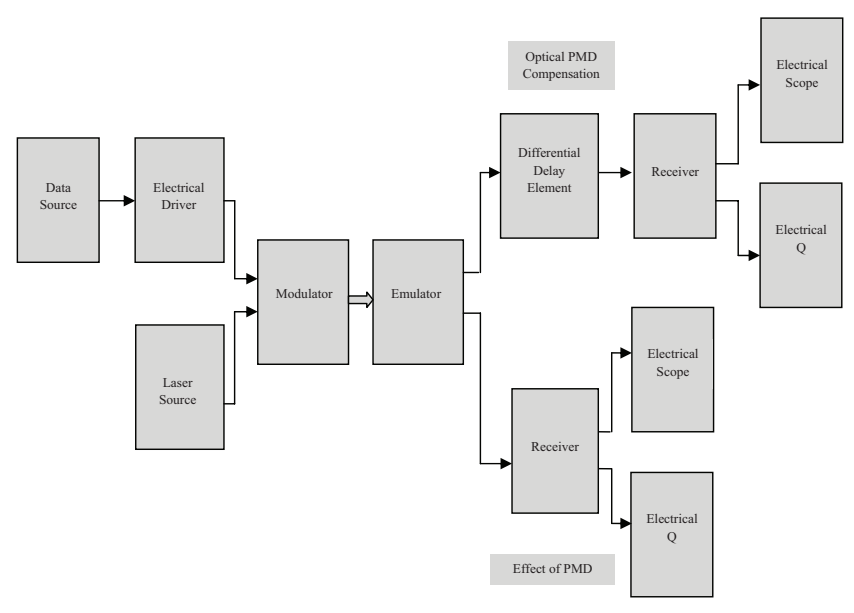

Figure 3. Simulation block for analyzing effect and compensation of PMD. 
achieved which can be seen from the eye diagrams. The distorted signal is received by the receiver and is analyzed through eye diagrams. For analyzing the optical compensation the signal emulated by the emulator is passed through a deterministic differential group delay element which splits the signal into two and provides a determined delay to one of the signal.

\section{Simulation Results}

The effect of PMD in a fiber link and the compensation of PMD by using an optical compensation technique is simulated using OptSim 5.3 [8]. The effect of PMD in an optical fiber link and an optical compensation technique is analyzed through various eye diagrams by performing parametric run. A parametric run is performed to simulate the time varying property of the emulator [9]. The eye diagrams give the $\mathrm{Q}$ values at each run from which the results can be interpreted. The results of the simulations are shown below.

Figure 4 shows the eye diagram having a $\mathrm{Q}$ value of $11.473 \mathrm{~dB}$ at run1. The effect PMD is analyzed by performing a parametric run which shows the time varying property of PMD.

Figure 5 shows that the due to the time varying effect the eye diagram changes and thus $\mathrm{Q}$ value also changes.

Figure 6 shows that the variations in the eye diagram. Thus Figures 4 to 6 shows the effect of PMD in an optical fiber link of $100 \mathrm{~km}$.

Figures 7 to 9 shows the effect of optical compensation at 3 different runs by which the $Q$ value is increased.

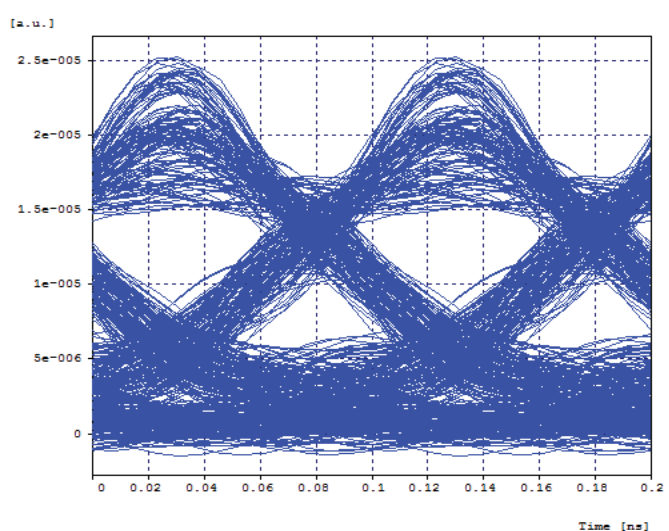

Figure 4. Eye diagram at run 1 showing a $Q$ value of 11.473 dB.

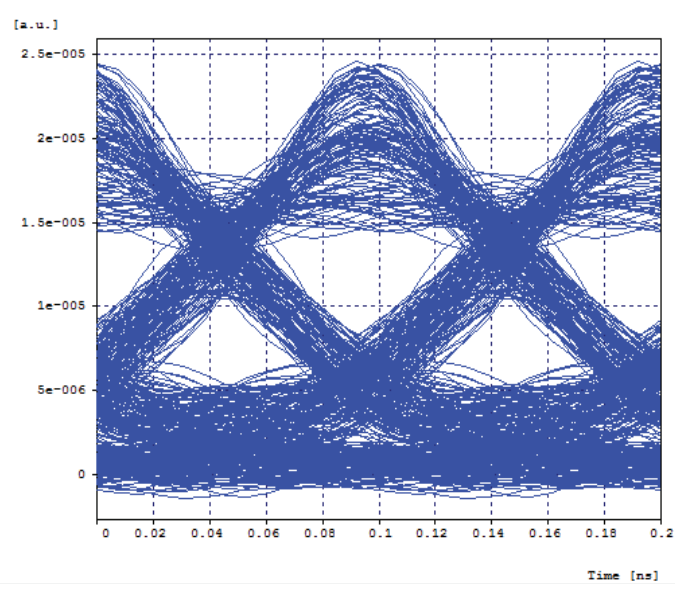

Figure 5. Eye diagram at run 2 showing a $Q$ value of 11.59 dB.

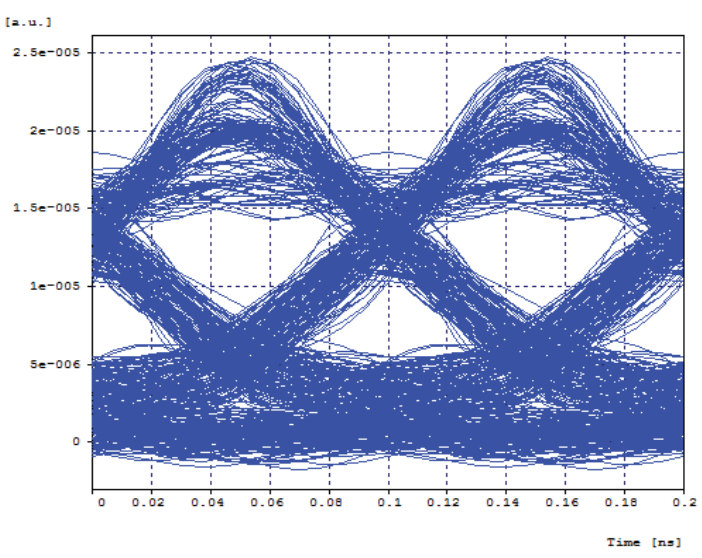

Figure 6. Eye diagram at run 3 showing a $Q$ value of 11.585 dB.

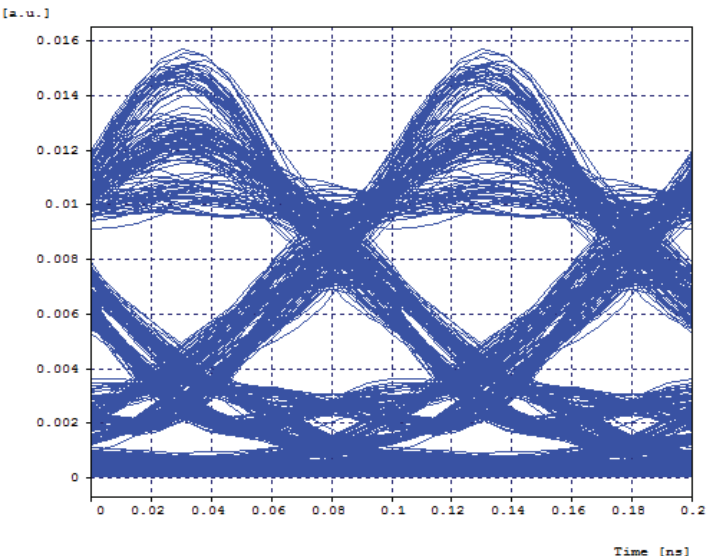

Figure 7. Eye diagram at run 1 showing a $Q$ value of 12.427 $\mathrm{dB}$. 


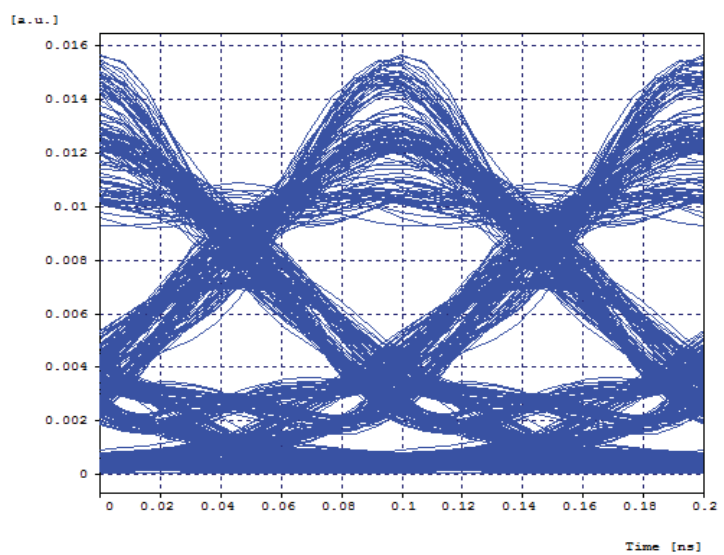

Figure 8. Eye diagram at run 2 showing a Q value of 12.579 $\mathrm{dB}$.

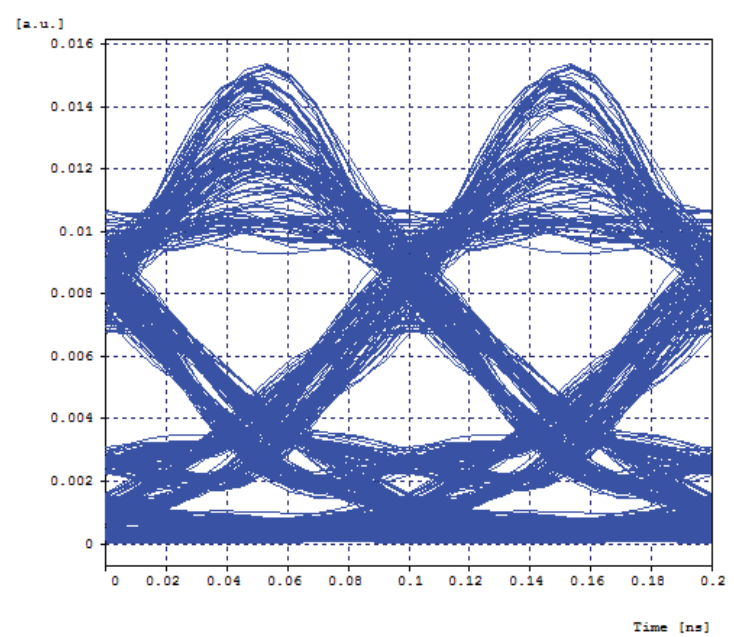

Figure 9. Eye diagram at run 3 showing a Q value of 12.749 $\mathrm{dB}$.

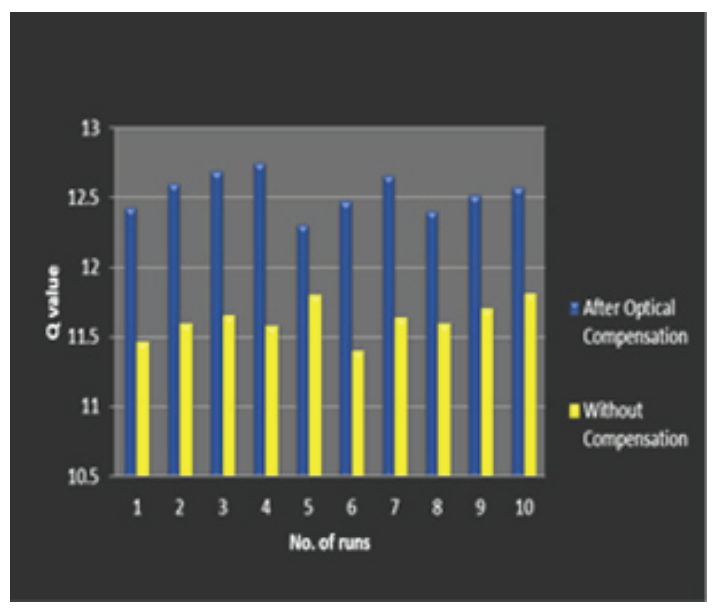

Figure 10. Comparison of $Q$ values without and after compensation of PMD.
Here the above Figure 10 shows the comparison of $\mathrm{Q}$ values obtained without any compensation and after the proposed optical compensation technique.

\section{Conclusion}

The paper is based on the analysis and optical compensation of polarization mode dispersion. This is a time changing quantity which causes a serious problem that limits distances. The polarization mode dispersion in a fiber link is analyzed through various eye diagrams by parametric run in OptSim simulation which confirms the degradation of system performance at higher bit rates. An optical compensation method by which the effect of PMD can be mitigated is shown as an increase in $\mathrm{Q}$ value and decrease in BER from the eye diagram of the simulation.

\section{Acknowledgement}

The author wish to thank the "Department of Electronics and Communication Engineering", TKM Institute of Technology for providing the facility to complete this work.

\section{References}

1. Borzycki K (2012). Temperature dependence of polarization mode dispersion in tight-buffered optical fibers, Journal of Telecommunications and Information Technology, 56-66.

2. Kogelnik H, R M Jopson et al. (2006). Polarization-mode dispersion, Chapter 15, Optical Fiber Telecommunications, vol IV B, Kaminow I P, Li T (Eds.), Academic Press, San Diego, CA.

3. Agarkar A M, Joharapurkar P (2010). PMD \& DGD performance analysis in SMF due to fiber irregularities, International Journal of Computer Applications, vol 12, No. 6.

4. Jamaludin M, Abas A F et al. (2005). Issues on polarization mode dispersion (PMD) for high speed fiber optics transmission, Suranaree Journal of Science and Technology, vol 12(2), 98-106.

5. Kashyap K, and Singh H (2010). Study the effects and compensation of polarization mode dispersion at different bit rates, IOSR Journal of Engineering, vol 2(7), 32-40.

6. Bülow H, Xie C et al. (2009). PMD Compensation/ Mitigation Techniques for High-Speed Optical Transport, 
Wiley Periodicals, Bell Labs Technical Journal, vol 14(1), 105-124.

7. Manohari R G, and Sabapathi T (2011). Analysis and reduction of polarization mode dispersionin an optical fiber, International Conference on Recent Advancements in Electrical, Electronics and Control Engineering.
8. Waddy D S, Chen L et al. (2010). A novel dynamical polarization mode dispersion emulator, IEEE Photonics Technology Letters, vol 1, No. 8, 534-536.

9. Chauvel G (2008). Anritsu Corporation, Dispersion in Optical Fiber, vol 11, No.4, 12. 